\title{
THE Asterias rubens complement system: Comparisons with lower vertebrates
}

\author{
Michel Leclerc ${ }^{*}$ and Nicolas Kresdorn ${ }^{2}$ \\ ${ }^{1} 556$ rue Isabelle Romée, 45640 Sandillon, France \\ ${ }^{2}$ GenXpro, Frankfurt, Germany
}

\begin{abstract}
Seven complement components have been discovered in 2013. Another component: the C6 component was found in sea star, when compared to rainbow trout genome.
\end{abstract}

\section{Introduction}

We have recently described the "Sea star complement Evidence" [1]. We remarked that C6 and C7 components were missing in sea star transcriptome when compared to mouse one.

An extensive study allowed us to research these components in less evolved animals (phylogenetically speaking) than mouse. Genomic features of the rainbow trout: Oncorhynchus mykiss have helped us, in this study.

At this point, we were attempting to determine how many similar complement components might be present in Asterias rubens (Invertebrate) and in Oncorhynchus mykiss (Vertebrate).

\section{Materials and methods}

Sea stars Asterias rubens were obtained from the Biology Institute (Gothenbugh University) Immunizations, genomic studies were already described [1]. After ligation of adapters for Illumina's GSII sequencing system, the cDNA was sequenced on the Illumina GSII platform sequencing.

$1.100 \mathrm{bp}$ from one side of the approximately $200 \mathrm{bp}$ fragments. Sequences were assembled using Velvet [2].

\section{Results}

Three complement components: C1r, C4, C1 inhibitor of the classical activation pathway have been fully sequenced in rainbow trout [3].

C6 was discovered in trout in 2006 [4].

Sea star C1q subunits A, B, C, were sequenced in A. Rubens [1].

$\mathrm{C} 2, \mathrm{C} 4 \mathrm{~B}$, and $\mathrm{C} 3$ which is central in mammals to both the classical and alternative pathways, $\mathrm{C}$,

C5, C8 were also sequenced (1) in Asterias rubens

As for C6, it was shown as following, when compared to Oncorhynchus mykiss genome:

One contig (Contig11285|m.9708) could be annotated via BLASTX to Oncorhynchus mykiss "Complement component C6" from the Trembl database, with an e-value of 3.75e-13. On an aligned region of 113 amino acids, 37 positive and 56 identical amino acids were found.

T C G A C A C T T A C A A A A A G C A T C T C A A C C C G A G T A G G A A G G A A T C T C T T T T A G T T G C A G T A A A T T T T G A A T T T G T A T A A T T C A G T A T T T T G T G C T C C C T T T G G T A T C A G T T T A G A T C C A C A C A A C C T G T G A A A A A C T T C A G T A C T T A C T A G A T T T C G C C A A C G C A A C G G T A A A C G A G T C A T T T G A T T T T G A C C A T C A T C A A C T GAAGCA A C GCACGT A A T A C A C A C A A C A A A C G G A A C A T T T T G T G T G T A G T T T C C A G C G A T T CGA GA A GCA A A T C A A A G A C A A G A T G T C T T T A C C C A G T G A T G T T G A A A C A G A C T C C G T C A T G GA T A G T C C A G C A G A G A T T C A T A T GA A C A T G A A T A A G C T A C A A T C T A A A C T T C C C A G C G T TA C T CA A GA C GA GA GA T T T GA C T C C G GA A T T G A C T C G T T A C G T T C T G T T G A T T C G G C G T A C T G C T T G A G C T T C G A A A G G G A A T C G A G C C T G G C T T C G A T A A A T G A G A A G A C G T C T C T C A C A TC A C A C C T G C A A C A G C T C C A T C T T T C A C A T G A A A C A A G A A C A G A A A C C G A G A A G C T G A A A C G A C A G T A G A A G A C A T C GA T GA A G C T T A T C A T GA T GA G T G T A C T A T G T C T G A A A C A C T CGA C A A T T T G G A A G A A A C T G C A A G A A T T G T G G A A T A T C C T G A A C A A A G A T G C A C G G G A C G T C T T A C A G A T G A T G C C T T C G A C C A A G A C C A A G A G G G A G A T A C G C C C C T T C A T C T T G C T A T T A T T CA T A A G GA A G T GGACT T C GCA GA A A A T T C A T C A T C T T T G T T G CA GA T C C T GA G T T A C T G A A C A T C A G C A A T G A T C T T A T G C A G A C T C

Correspondence to: Michel Leclerc, 556 rue Isabelle Romée, 45640 Sandillon, France, E-mail: mleclerc45@gmail.com

Received: January 16, 2016; Accepted: February 25, 2016; Published: February 29,2016 
C T T T A C A C C T T A G C G T A T T A A C A A G G C A A C A A GA T A T C T G T C G T G T T C T C G T C T T G G G C A A T G C C C A A A T C G A C T G C A C C G A C C G A A A C G G C G A C A C T C C T C T T C A T A T T G C A T G C A G A C T G A G A G A T G A G G G C T G T A T C A G A G C T C T G A C T GA A G G A A T A T C T C C A C T C G A G C G T A A G A GA GGGA TGGT T C C A C A GA A T A GA GCA A G T GGGG T A C A A C A G C T T C C A C A G A A T C T T G A A C T C A G A A A C T T T G A A G G C T A C A C A T G C A T C C A T A T T G C A G G A T T C G C T T G T A GCG T C G A T C A G T T G G A G T A C C T T G T G C A G C T A G G C G G C GA C A T A A A T G C C C C G GA T G G A A A A G C G G A A G G A C C A T T C T C C A C T A C G C T G T A G A G G C GGG T GA C T T T T C T C T T T G T C A G T A C C T C A T T G CGAACTTGGGTGCCAATGTTAATGCGTTGACCTTTGACCAGTGCACACCC3'

\section{C7 was not found.}

\section{Discussion and conclusion}

The sea star A. rubens, although considered to be more primitive than lower vertebrates (as trout) seems to have evolved much more sophisticated immune defense mechanisms.

We find much more complement components in the sea star than in trout: 8 out of 9 , when compared to mouse. How do we explain these differences between trout and $A$. rubens?

Phylogenetically speaking the sea star could be situated in "an evolutive cul de sac" and might evolved more quickly than rainbow trout, in term of innate immunity.

As for adaptative immunity, rainbow trout is more evolved [5] than
Asterias rubens which presents an "invertebrate primitive antibody" in response to antigenic injury [6].

This review has described a rather rich catalogue of immune factors in sea star and trout that serve as potent molecules in the defense of these animals against environmental threats.

Taken together one cannot come away with any conclusion other than sea stars have developed a very impressive set of mechanisms to deal with environmental threats.

The same logic would apply to an explanation of why the sea star A.rubens has evolved the ability to develop innate and adaptative immunity.

Further studies are bound to unravel the mystery and add to the above information to give a clearly picture of the sequence of events.

\section{References}

1. Leclerc M, Kresdorn N, Rotter B (2013) Evidence of complement genes in the sea-star Asterias rubens. Comparisons with the sea urchin. Immunol Lett 151: 68-70. [Crossref]

2. Zerbino DR, Birney E (2008) Velvet: algorithms for de novo short read assembly using de Bruijn graphs. Genome Res 18: 821-829. [Crossref]

3. Wang T, Secombes CJ (2003) Complete sequencing and expression of three complement components, $\mathrm{C} 1 \mathrm{r}, \mathrm{C} 4$ and $\mathrm{C} 1$ inhibitor, of the classical activation pathway of the complement system in rainbow trout Oncorhynchus mykiss. Immunogenetics 55: 615-628. [Crossref]

4. Chondrou MP, Mastellos D, Zarkadis IK (2006) cDNA cloning and phylogenetic analysis of the sixth complement component in rainbow trout. Mol Immunol 43: 10807. [Crossref]

5. Partula S, Schwager J, Timmusk S, Pilström L, Charlemagne J (1996) A second immunoglobulin light chain isotype in the rainbow trout. Immunogenetics 45: 44-51. [Crossref]

6. Vincent N, Osteras M, Otten P, Leclerc M (2014) A new gene in A. rubens: A sea star Ig kappa gene. Meta Gene 2: 320-322. [Crossref]

Copyright: (C2016 Leclerc M. This is an open-access article distributed under the terms of the Creative Commons Attribution License, which permits unrestricted use, distribution, and reproduction in any medium, provided the original author and source are credited. 